\title{
Asymptotic Coefficients of Hermite Function Series
}

\author{
JOHN P. BOYD \\ Department of Atmospheric and Oceanic Science, \\ University of Michigan, Ann Arbor, Michigan 48109
}

Received March 30, 1983; revised August 5, 1983

\begin{abstract}
By using complex variable methods (steepest descent and residues) to asymptotically evaluate the coefficient integrals, the numerical analysis of Hermite function series is discussed. There are striking similarities and differences with the author's earlier work on Chebyshev polynomial methods (J. Comp. Phys. 45 (1982), 45-49) for infinite or semi-infinite domains. Like Chebyshev series, the Hermite coefficients are asymptotically given by the sum of two types of terms: (i) stationary point (steepest descent) contributions and (ii) residues at the poles of $f(z)$, the function being expanded as a Hermite series. The stationary point term is determined solely by the asymptotic behavior of $f(z)$, i.e., how rapidly $f(z)$ decays as . $z \rightarrow \infty$ along the real axis. Unlike Chebyshev series, however, it is necessary to perform a separate analysis for functions which decay faster or slower than the Gaussian function $\exp \left[-A z^{2}\right]$. Singular functions, too, fall into two categories. Those that decay rapidly with $z$ have asymptotic Hermite coefficients which are dominated by the singularity, but functions which decay as slowly as $\operatorname{sech}(z)$ or slower have Hermite coefficients dominated by the stationary point terms, and the singularity is irrelevant. The end product of the analysis is the same as in the earlier work: simple, explicit formulas to optimize the efficiency of Hermite methods and estimate a priori how many degrees of freedom are needed, provided one knowns at least crudely: (i) the asymptotic behavior of $f(z)$ and (ii) its singularity nearest the real axis. Rather surprisingly, one finds Hermite functions superior to Chebyshev polynomials for some classes of functions when the computational domain is infinite.
\end{abstract}

\section{INTRODUCTION}

Recently, the author [1] applied the method of steepest descent to obtain the asymptotic Chebyshev polynomial coefficients for different ways of representing functions on an infinite or semi-infinite interval. This made it possible to determine (i) which technique, domain truncation or mapping, was most efficient for a given class of function and (ii) how to choose the optimum domain size or mapping parameter.

The goal of this present work is to explore a third alternative for an infinite domain: Hermite function series. Although the mechanics of Hermite spectral methods are slightly more complicated than for Chebyshev polynomials, there is a very close parallel between them. The use of Hermite functions is particularly appealing because they are often the exact unperturbed eigenfunctions (examples: the harmonic oscillator in quantum mechanics and equatorial waves in dynamic meteorology and oceanography) or the limiting asymptotic eigenfunctions (Mathieu functions, prolate spheroidal wave functions) for many problems of physical interest. 
The end products of this investigation are simple asymptotic formulas for the Hermite coefficients for general functions. It is only necessary to know (i) how rapidly the function decays along the real axis and (ii) the location of its singularity nearest the real axis, and the rest is trivial.

There is a purely mathematical fascination with seeing how it all works out, with understanding what determines the rate at which the series converges. Mathematicians have been intensively studying orthogonal polynomials for over a century. Much is known about exotic polynomials and about exotic means of summing the poorly converging series of the familiar polynomials when the function being expanded is discontinuous or otherwise pathological, but little attention has been paid to the rate of convergence of the series for smooth, non-pathological functions-such as usually arise in engineering and physics problems--though this is the only question of interest to a numerical analyst. The present work thus fills a significant gap in the existing theory of Hermite functions.

From the viewpoint of numerical analysis, these asymptotic formulas are useful in solving differential equations using spectral or pseudospectral methods with Hermite functions as the basis functions. The philosophy is the same as in the author's earlier work [1]: The analysis is limited to asymptotic formulas for the Hermite coefficients of a known function $f(z)$, and no differential equations are actually solved here. This would seem to be rather restrictive, but it is well known that the errors in approximately calculating Hermite coefficients via some algorithm for solving a differential equation are of the same order of magnitude as those made by truncating the exact Hermite series of the exact solution after the same number of terms. In other words, the best way to obtain an accurate solution $f(z)$ to a differential equation as an approximate sum of $N$ Hermite functions is to adjust the parameters of the problem so that the $N+1$ st and higher Hermite coefficients of $f(z)$ are as small as possible.

How can one make this adjustment when $f(z)$ is unknown? The answer is that the formulas derived here depend only upon the asymptotic behavior of $f(z)$. The asymptotic behavior of the solution to a differential equation can usually be obtained-by the WKB method, for example-without explicitly computing an exact, numerical solution to the differential equation first. It will be assumed that the reader can obtain descriptions of how to use series of orthogonal functions, which include Hermite functions as a particular case, to solve differential equations from works like that by Gottlieb and Orszag [2]. The focus here is not on the algorithms, but rather on their numerical analysis, i.e., on how well the algorithms work and how one can optimize their efficiency.

The plan of the paper is as follows. Section 2 gives some basic definitions and reviews what is already known about Hermite series from the many papers of Einar Hille [3-6] and an earlier work of the author's [7]. It is necessary to separately consider functions which decay more rapidly than a Gaussian ("super-Gaussian") or more slowly than a Gaussian ("sub-Gaussian" such as $\operatorname{sech}(z)$ ), so Sections 3 and 4 deal with the former while Section 5 discusses the latter. Section 3 derives the "regular" asymptotic Hermite coefficients in which the width of the functions is kept fixed as one takes the limit $n \rightarrow \infty$. To obtain best results with a given finite number 
of Hermite functions, however, it is necessary to vary the width of the Hermite functions relative to that of $f(z)$, the function being expanded, as $N$, the number of Hermite functions kept in the truncation, is varied. Section 4 therefore derives the "uniform" asymptotics for "super-Gaussians" in which both $N$ and the width are increasing to infinity together.

Section 5 derives the "regular" asymptotic coefficients for "sub-Gaussian" functions. The "uniform" asymptotics are not derived here, however, because the author has not yet figured out how to do it.

For singular functions, Section 6 shows that there are two contributions: (i) a steepest descent term and (ii) a residue at the pole of $f(z)$. Section 6 evaluates the residue term; the steepest descent contribution is the same as for entire functions with the same rate of decay along the real axis and is evaluated in Sects. 3, 4, and 5 for the various cases.

The emphasis on asymptotic results, i.e., formulas for large numbers of Hermite functions, can be misleading because it is often possible to use Hermite methods effectively even with very low truncations to obtain analytical as opposed to numerical results. (Recall the remark made earlier: A single Hermite function is a good approximate solution in the appropriate parameter regime for many problems of physical interest.) Section 7 provides a simple example: An approximate, analytic solution to the quartic anharmonic oscillator of quantum mechanics is obtained by using Galerkin's method with just two Hermite functions and solving the resulting quadratic equation for the eigenvalue. The perturbation series for this case is only asymptotic and is ineffective except for very weak perturbations, but the $N=2$ Hermite spectral solution is accurate even for moderately large values of the coupling constant.

The final section is a summary and prospectus.

\section{Basic Properties of Hermite functions}

The normalized Hermite functions $h_{n}(z)$ are defined by

$$
h_{n}(z) \equiv\left(2^{n} n ! \pi^{1 / 2}\right)^{-1 / 2} e^{-(1 / 2) z^{2}} H_{n}(z)
$$

where the $H_{n}(z)$ are the usual (unnormalized) Hermite polynomials. The normalized Hermite functions satisfy the bound [8]

$$
\left|h_{n}(z)\right| \leqslant 0.816 \quad \text { for all } n \text { and all real } z
$$

This is a fairly tight bound in the sense that (5.2) below shows that (2.2) overestimates $\max \left|h_{n}(z)\right|$ by less than a factor of two for all $n<216$. This bound in turn implies

$$
\left|f(z)-\sum_{n=0}^{N} a_{n} H_{n}(z)\right| \leqslant 0.816 \sum_{n=N+1}\left|a_{n}\right|
$$


for all real $z$, where

$$
a_{n}=\int_{-\infty}^{\infty} f(z) h_{n}(z) d z
$$

is the $n$th coefficient in the Hermite series of $f(z)$. Equation (2.3), which in words states that the error in truncating a Hermite series after $N$ terms is bounded by the sum of the absolute values of all the neglected coefficients, is extremely useful. Normally, of course, one does not know the neglected coefficients exactly, but if one knows the asymptotic form of the coefficients-as will be derived in the rest of the paper-then (2.6) can be approximately evaluated to give a fairly tight bound on the truncation error. For smooth functions, the exponential decrease of $\left|a_{n}\right|$ with $n$ implies the infinite sum in (2.3) is dominated by the first term so that

$$
\left|f(z)-\sum_{n=0}^{N} a_{n} H_{n}(z)\right| \sim O\left(a_{N}\right)
$$

where, to be conservative (and also to be consistent with the truncation error $E_{S}(N)$ as defined in [1]) $a_{N+1}$ has been replaced by $a_{N}$. Thus, the problem of bounding the errors in a truncated Hermite series reduces to that of simply estimating the coefficients for large $N$.

The simplest bound on the Hermite coefficients is provided by the following.

THEOREM. If $f(z)$ is such that $d^{j} f / d z^{j}(z)$ and $z^{j} f(z)$ (and all lower derivatives) are bounded and integrable on $[-\infty, \infty]$ for all real $z$, then

$$
\lim _{n \rightarrow \infty} n^{j / 2}\left|a_{n}\right|=d
$$

where $d$ is a constant independent of $n$. If $f(z)$ is infinitely differentiable and decays exponentially fast, i.e., faster than any finite inverse power of $z$, as $|z| \rightarrow \infty$ for real $z$, then the $\left|a_{n}\right|$ decrease faster than any finite inverse power of $n$ and the series is said to possess the property of "exponential" or "infinite order" convergence. The condition that $z^{j} f(z)$ be integrable demands that $f(z)$ decay at least as fast as $1 / z^{j+1+\varepsilon}$, where $\varepsilon>0$.

Proof. Repeated integration-by-parts of the coefficient integral (2.4) using the indefinite integral

$$
\int^{z} \bar{H}(z) d z=\frac{\bar{H}_{n+1}(z)}{(2 n+2)^{1 / 2}}
$$

where $\bar{H}_{n}(z)$ is the $n$th normalized Hermite polynomial is all that is needed. For example, one integration-by-parts gives 


$$
\begin{aligned}
a_{n}= & \left.\frac{f(z) e^{-(1 / 2) z^{2}} \bar{H}_{n+1}(z)}{(2 n+2)^{1 / 2}}\right|_{-\infty} ^{\infty} \\
& -\frac{1}{(2 n+2)^{1 / 2}} \int_{-\infty}^{\infty}\left[\frac{d f}{d z}-z f(z)\right] \bar{H}_{n+1}(z) e^{-(1 / 2) z^{2}} d z
\end{aligned}
$$

If $f(z)$ is merely bounded at infinity, then the $\exp \left[-(1 / 2) z^{2}\right]$ factor will ensure that the boundary term will vanish, leaving the integral in (2.8). Since it is assumed

$$
\int_{-\infty}^{\infty}\left[\frac{d f}{d z}-z f(z)\right] d z=M
$$

where $M$ is a finite constant-this is what is meant by "integrable on $[-\infty, \infty]$ " in the theorem-and since the Hermite functions satisfy the bound (2.2) which is independent of $n$, it follows (by replacing $\bar{H}_{n+1}(z) e^{-(1 / 2) z^{2}}$ in $(2.8)$ by its bound $0.816)$ that

$$
\left|a_{n}\right| \leqslant \frac{0.816 M}{(2 n+2)^{1 / 2}}
$$

which proves the theorem for $j=1$. A second integration-by-parts shows $a_{n} \sim O\left(n^{-1}\right)$, a third that $a_{n} \sim O\left(n^{-3 / 2}\right)$ and so on. If $f(z)$ is infinitely differentiable and exponentially decaying as $|z| \rightarrow \infty$, then one can integrate-by-parts an arbitrary number of times, which implies $a_{n}$ decreases faster than any finite inverse power of $n$. If, on the other hand, some finite derivative of $f(z)$ is unbounded, then one can integrate-by-parts only a finite number of times and there will exist a maximum value of $j$, not necessarily an integer or half-integer, such that $\lim _{n \rightarrow \infty} n^{j}\left|a_{n}\right|$ is finite. (This maximum is the "algebraic index of convergence.")

This theorem is not the sharpest possible, but it will suffice for present purposes because most solutions to problems in physics and geophysics do not exhibit jump singularities or other pathologies but instead are infinitely differentiable. (Shocks and caustics are exceptions, of course, but usually require special treatment anyway.) The rest of this paper (except Sect. 7) will therefore deal only with exponential convergence.

This Hermite theorem is a close parallel of others known and used for many years with Fourier series and Chebyshev polynomials [2], but it has not been explicitly given before to the author's knowledge. It is striking that each integration-by-parts for Fourier and Chebyshev functions brings in a factor of $1 / n$ while for Hermite only a factor of $1 / \sqrt{n}$. We will explain why at the end of the section.

Two definitions already employed in [1] will also be useful here.

DEFInItion. The exponential index of convergence $r$ of a sequence $\left\{a_{n}\right\}$ is

$$
\lim _{n \rightarrow \infty} \frac{\log \left|\log \left(\left|a_{n}\right|\right)\right|}{\log (n)}=r
$$


equivalently, it is the least upper bound $r$ of those $\beta$ for which

$$
a_{n} \sim O\left(e^{-p n^{B}}\right)
$$

for some constant $p$ as $n \rightarrow \infty$.

In Boyd [1], (2.11) was used and the exponential convergence index was called the "exponential convergence order." Substituting "index" for "order" in (2.11)-(2.12) seems preferable because "order" has been traditionally used to describe the behavior of $f(z)$ itself as in the definition of the "order" of an entire function. The motive for defining the exponential index of convergence is that $a_{n}=\exp \left[-n^{r}\right]$ has "exponential convergence" for any $r>0$, but in practice, it is obviously very important whether $r=0.001$ or $r=1.0$. The reason for the nested logarithms in (2.11) and the "least upper bound" in (2.12) is to accomodate forms like $a_{n} \sim n \exp \left[-n^{1 / 2}\right]$ which have an algebraic function of $n$ multiplying the exponential. As in [1], such algebraic factors of $n$ will usually be ignored because they are only of secondary importance in choosing the best spectral algorithm for a given problem.

Definition. The order of real axis decay $k$ is the least upper bound of $j$ for which

$$
f(z) \sim O\left(e^{-p|z| j}\right)
$$

for some constant $p$ as $|z| \rightarrow \infty$ along the real axis. The function is said to be "subGaussian" or "super-Gaussian," respectively, if

$$
k<2 \quad \text { ["sub-Gaussian"] }
$$

or

$$
k>2 \quad \text { ["super-Gaussian"] }
$$

In practice, the order of real axis decay is, for entire functions, equal to the "order" of the entire function as it is usually defined. One can, however, contrive examples like $f(x)=\cos \left(z^{6}\right) \exp \left(-z^{4}\right)$ which has entire function order $\rho=6$ but real axis decay order $k=4$. In the rest of this paper, it will be implicitly assumed that $\rho=k$ although the extension to $\rho \neq k$ is trivial.

Armed with these concepts, it is possible to give the following summary of what was previously known about the convergence of Hermite function series:

(i) (Hille [3]) The domain of convergence of a Hermite series is the infinite strip about the real axis bounded by the lines

$$
\operatorname{Im}(z)= \pm w
$$


(ii) (Hille [4]) The width $w$ is

$$
w=\left\{\begin{array}{cl}
\infty & {[\text { entire functions, } k>1]} \\
\tau & {[\text { singular functions, } k>1]} \\
\min [p, \tau] & {[\text { singular functions, } k=1]} \\
0 & \left\{\begin{array}{l}
\text { [entire functions, } k<1] \\
{[\text { singular functions, } k<1]} \\
{[\text { functions with finite algebraic }} \\
\text { index of convergence }]
\end{array}\right.
\end{array}\right.
$$

where $\tau>0$ is the perpendicular distance from the real axis of that singularity of $f(x)$ which is nearest the real axis and where $p$ is the constant in the asymptotic behavior $f(z) \sim O\left([] e^{-p|z|}\right)$, where [ ] denotes irrelevant algebraic factors of $z$. A width of 0 means that the Hermite series converges to $f(z)$ only on the real $z$ axis. It is noteworthy that $k$, the order of real axis convergence, is dominant over singularities of $f(z)$ [except those on the real axis] when $k<1$; this is never true for power series convergence which is why the concept of "order" has been applied only to entire functions in the past. and

(iii) (Hille [4]) If the width $w$ is finite, the exponential convergence index $r=\frac{1}{2}$

$$
a_{n} \sim O\left(A(n) e^{-w(2 n+1)^{1 / 2}}\right)
$$

where $A(n)$ is a previously unknown algebraic factor of $n$ which is computed for the first time in Sect. 6 (and shown to be $O\left(n^{-1 / 4}\right)$ ).

(iv) (Boyd [7]) For entire functions

$$
\begin{array}{ll}
r \leqslant \frac{k}{2(k-1)} & \text { ("super-Gaussian," } k>2 \text { ) } \\
r \leqslant \frac{k}{2} & \text { ("sub-Gaussian," } k<2 \text { ) }
\end{array}
$$

The difference between (2.19) and (2.20) strongly suggests that different treatments are needed for the "sub-Gaussian" and "super-Gaussian" cases; this is indeed true, so these classes will be discussed in separate sections.

Turning from convergence rate to the Hermite functions themselves, the $h_{n}(z)$ oscillate between the turning points

$$
\left|z_{t}\right|= \pm \sqrt{2 n+1}
$$


and decay exponentially as $\exp \left(-[1 / 2] z^{2}\right)$ for $|z|>\left|z_{t}\right|$. For small values of $z$, one can use the ordinary asymptotic expansions

$$
h_{n}(z) \sim \frac{(-1)^{n} \sqrt{(2 n) !}}{\pi^{1 / 4} 2^{n} n !} \cos \left[(2 n+1)^{1 / 2} z-n \pi / 2\right]
$$

for $n$ even and a similar expression involving the sine function for $n$ odd. When $|z|$ is $O(\sqrt{2 n+1})$, however, one must replace $(2.22)$ by more accurate approximations as done in Sections 4 and 5.

To carry out the method of steepest descents, it is necessary to decompose

$$
h_{n}=h_{n}^{+}+h_{n}^{-}
$$

where

$$
h_{n}^{\perp} \sim \frac{e^{ \pm i(2 n+1)^{1 / 2 z}}}{\pi^{1 / 2} 2 n^{1 / 4}}
$$

for even $n$ and the same multiplied by $\pm i$ for odd $n$ and we have employed the asymptotic form of the factorials in (2.22). G. N. Watson [9] shows that the $h_{n}^{ \pm}(z)$ can be written without approximation in terms of parabolic cylinder functions of complex argument and Hille [4] gives their WKB approximations.

These asymptotic forms explain much of the difference between the convergence rate of Hermite functions on the one hand and Fourier and Chebeyshev series on the other; specifically, why each additional order of differentiability improves Fourier convergence by $n^{-1}$ but Hermite only by $n^{-1 / 2}$ and also why the Fourier coefficients of a singular function are $O\left(e^{-p n}\right)$ while the Hermitc coefficients decreasc only as $O\left(e^{-q n^{1 / 2}}\right)$ for some constants $p$ and $q$. The reason is that Hermite functions must vary in two competing ways as $n$ increases. First, they must oscillate more and more rapidly with $z$ to provide increased resolution near the origin. Second, the width of the region of oscillation must increase with $n$ because the Hermite functions must resolve the whole real axis as $n \rightarrow \infty$. The result is that whereas Fourier terms $\cos (n x)$ and $\sin (n x)$ have wavelengths proportional to $1 / n$, Hermite functions have local wavelengths proportional to $1 / \sqrt{n}$ (see (2.22)) because the interval between the turning points is increasing as $\sqrt{n}$ also as shown by (2.21). Because the Hermite series must serve two masters, $\sqrt{n}$ must replace $n$ in the Hermite analogues of convergence theorems for Fourier and Chebyshev series on a fixed, finite interval.

Naively, one might suppose that Chebyshev expansions would always be superior to Hermite functions. Boyd [1] shows, however, that when Chebyshev polynomials are applied to an infinite interval, one must rescale the width of the Chebyshev polynomials to mimic the natural expansion-of-oscillation-interval-with-the-truncation which is built into the Hermite functions. 


\section{Regular Asymptotic Coefficients for "Super-Gaussians"}

For simplicity, consistent with the philosophy of Boyd [1], the application of the steepest descent method to evaluate the Hermite coefficients $a_{n}$ as $n \rightarrow \infty$ will be initially confined to model functions of the form

$$
f(z)=e^{-A z^{k}}
$$

where $k$ is an even integer greater than 2 . This seems terribly restrictive, but is made only to postpone a discussion of some fussy technical details whose solution is almost obvious once one has seen how it goes for functions like (3.1). The method is actually quite general and will ultimately bear fruit for functions with singularities, with asymptotic forms like (3.1) but of non-integral $k$, and for functions in which the exponential in (3.1) is multiplied by more slowly varying exponential or algebraic factors of $z$.

The method of steepest descent applies to integrals of the form

$$
I(n)=\int_{c} g(z) e^{\phi(z, n)} d z
$$

where $c$ is some contour in the complex plane (which in our case is the whole real axis). The key step is to deform the contour of integration into a new "steepest descent" path such that the integral is dominated, as $n \rightarrow \infty$, by the contributions from the neighborhoods of the stationary points $z_{s}(n)$ which are defined to be the solutions of

$$
\frac{d \phi\left(z_{s}, n\right)}{d z}=0
$$

Then as $n \rightarrow \infty$, the integral is approximately

$$
I(n) \sim \sum \sqrt{\frac{2 \pi}{-\phi^{\prime \prime}\left(z_{s}, n\right)}} e^{\phi\left(z_{s}, n\right)} g\left(z_{s}\right)
$$

where the sum is over all the stationary points on the new contour of integration and where the double prime denotes the second derivative with respect to $z$.

The stationary points are functions of $n$; if $\left|z_{s}(n)\right| \rightarrow \infty$ as $n \rightarrow \infty$, then it is legitimate to approximate $f(z)$ by its asymptotic approximation (3.1) in evaluating (3.3) and (3.4). If the stationary points grow more slowly with $n$ than the turning points $z_{t}$ of the Hermite functions, then it is equally legitimate to replace the Hermite functions by their asymptotic approximations (2.22), (2.24). In order to put the integral into the form of (3.2), however, it is necessary to replace the usual Hermite functions $h_{n}(z)$, which asymptotically are sines and cosines, by the sum of $h_{n}^{+}(z)$ and $h_{n}^{-}(z)$ whose asymptotics are complex exponentials. Thus, 


$$
\begin{aligned}
a_{n} & \equiv \int_{-\infty}^{\infty}(z) h_{n}(z) d z \\
& =I_{n}^{+}+I_{n}^{-}
\end{aligned}
$$

where

$$
\begin{aligned}
I_{n}^{ \pm} & =\int_{-\infty}^{\infty} f(z) h_{n}^{ \pm}(z) d z \\
& \sim \frac{g(n)}{\pi^{1 / 2} 2 n^{1 / 4}} \sum \frac{(2 \pi)^{1 / 2}}{\left(-\phi^{\prime \prime}\right)^{1 / 2}} e^{\phi}
\end{aligned}
$$

where

$$
g(n)=\left\{\begin{array}{cl}
1 & n \text { even } \\
\mp i & n \text { odd }
\end{array}\right.
$$

where the sum is over all stationary points on the steepest descent contour with

$$
\phi=-A z^{k} \pm i(2 n+1)^{1 / 2} z
$$

By explicit differentiation, the equation that determines the stationary points is

$$
-A k z^{k-1} \pm i(2 n+1)^{1 / 2}=0
$$

with the solution

$$
z=\mp e^{i \pi /(2 k-1])}\left[\frac{(2 n+1)^{1 / 2}}{k A}\right]^{1 /(k-1)}
$$

This has multiple solutions, but one can show by substituting (3.12) into (3.8) that the contributions from the pair nearest the real axis will be exponentially large in comparison to those from the other stationary points. The other stationary points may not be missed by the steepest descent contour, but the pair nearest the real axis lie in that half-plane where $h_{n}^{+}\left(h_{n}^{-}\right)$is decaying. The deformed contour will run along the real axis until $z$ is sufficiently small that the decay of $h_{n}^{+}(z)\left(h_{n}^{-}\right)$takes over and forces the contour into the upper (lower) half-plane to pass through the pair of stationary points with smallest imaginary parts. Fig. 1 shows the contours for $k=4$ and $k=6$.

The numerical values calculated here depend only on the local behavior of $f(z)$ and $h_{n}^{ \pm}(z)$ at the relevant stationary points, but there is an essential, implicit assumption: that a steepest descent contour equivalent to integration along the real axis exists. It is plausible that one always exists at least for single-valued $f(z)$, but the author is aware of no rigorous theory to prove this, so it will be left as an assumption. In specific cases, of course, the existence of the steepest descent contour can be proved by numerically calculating it as done to create Fig. 1. 

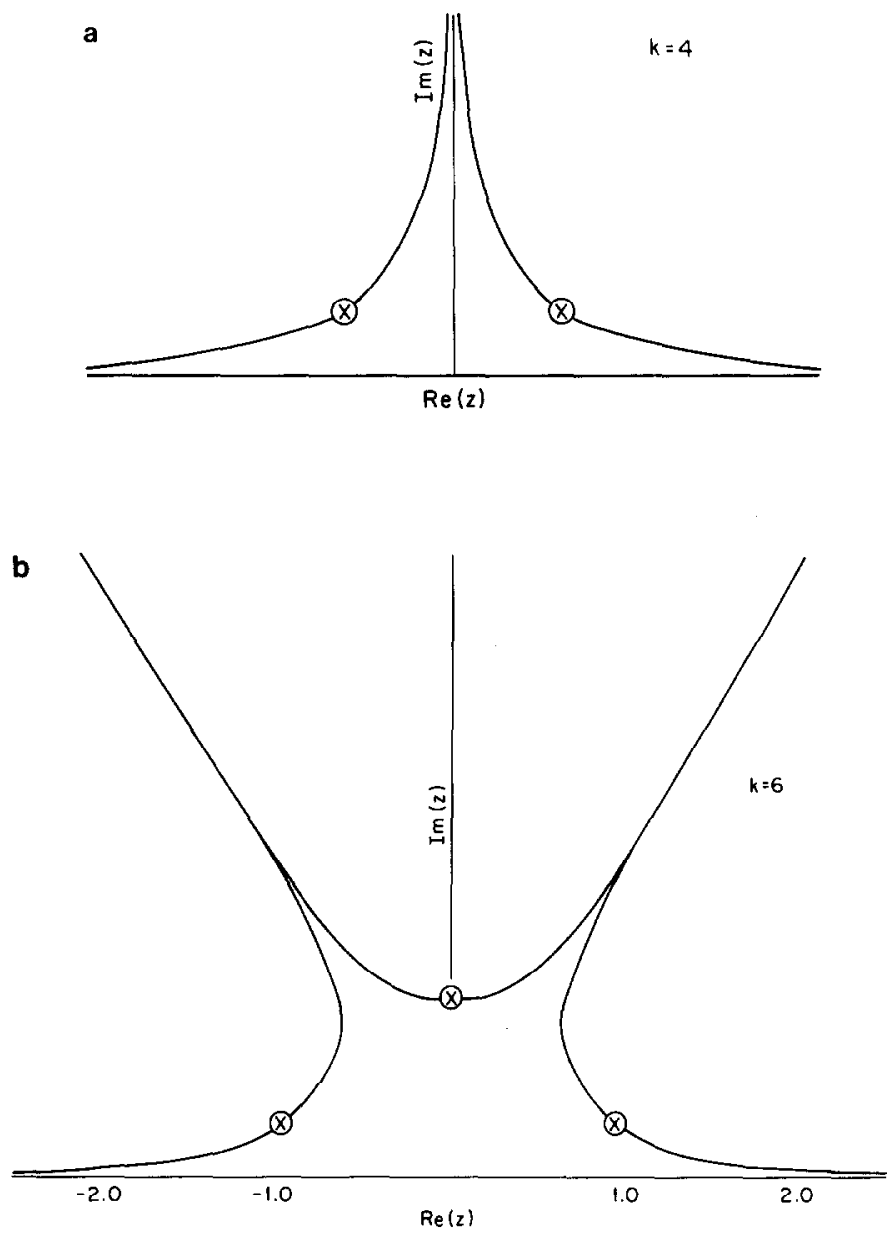

Fig. 1. Steepest descent paths for (a) $f(z) \exp \left[-A z^{4}\right]$ and (b) $\exp \left[-A z^{6}\right]$. Only the path for $h_{n}^{+}(z)$ is shown; that-for $h_{n}^{-}(z)$ is the reflection with respect to the real axis of the contour illustrated. The stationary points are marked by " $x$ " inside a circle. Note that the path for $\exp \left[-A z^{6}\right]$ passes through three stationary points, but the contribution from the stationary point on the imaginary axis is exponentially small in comparison to the contributions from the two stationary points closest to the real axis.

For a symmetric $f(z)$ such as the model functions (3.1), the contributions from the two stationary points in upper half-plane are complex conjugates and similarly for the stationary points in the lower half-plane. One finds as $n \rightarrow \infty$ with $A$ fixed

$$
a_{n} \sim \frac{1}{\pi n^{1 / 4}}\left(\frac{2}{R}\right)^{1 / 2} e^{-\sigma} \cos (\Omega-\theta / 2)
$$


where, letting $v=\sqrt{2 n+1}$

$$
\begin{aligned}
R & =v^{(k-2) /(k-1)}(k-1)(A k)^{1 /(k-1)} \\
\sigma & =v^{k /(k-1)}(k A)^{-1 /(k-1)}(k-1) \sin \left[\frac{\pi}{2(k-1)}\right] / k \\
\Omega & =v^{k /(k-1)}(k A)^{-1 /(k-1)}(k-1) \cos \left[\frac{\pi}{2(k-1)}\right] / k \\
\theta & =\frac{\pi}{2} \frac{(k-2)}{(k-1)}
\end{aligned}
$$

The form (3.13) is exact only for $f(z)=\exp \left[-A z^{k}\right]$ with $k$ an even integer. However, the analysis is the same for functions like $A i\left(z^{2}\right)$, which is asymptotically of the form (3.1) with $k=\frac{3}{2}$. The precise form of $f(z)$ may multiply (3.13) by a constant or by an algebraically varying factor of $n$, but examining the stationary point condition (3.11) and noting that $\left|z_{s}\right| \gg 1$ for $n \gg 1$, one sees that it is only the order of real axis decay $k$ which determines the dependence of $z_{s}$ on $n$. This in turn is sufficient to completely determine the exponential index of convergence which, recalling $v=(2 n+1)^{1 / 2}$, is from $(3.15)$

$$
r=\frac{k}{2(k-1)}
$$

This is the upper limit of the bound on the index of convergence for entire functions obtained by Boyd [7].

As noted in the introduction, one is normally interested in using Hermite functions to obtain an approximate series solution to a differential equation. Consequently, $f(z)$ is not known exactly anyway, so it is pointless to worry about the miscellaneous constants and fractional powers of $n$ embodied in $R, \Omega$, and $\theta$ in (3.13). One cannot hope to estimate $f(z)$ that closely beforehand. The exponential dependence upon $n$ as represented by $\sigma$ in (3.13) and by the index of convergence $r$ is a different story: Upon it hinges the success or failure of the Hermite spectral method. The point of this work is that if one can estimate $f(z)$ asymptotically-which is usually fairly easy-so as to obtain the order of real axis decay $k$, then the exponential index of convergence $r$ follows immediately from (3.18).

Equations (3.13) and (3.15) actually give information considerably more specific than $r$, but also imply a paradox: by rescaling $f(z)$ so as to make $A \rightarrow 0$, one can seemingly make the Hermite coefficients decrease arbitrarily fast. The resolution of this contradiction is the same as for the Chebyshev series on an unbounded interval discussed in Boyd [1]: For best results with a given number $N$ of Hermite functions, one must rescale $f(z)$ in an $N$-dependent manner. Unfortunately, this requires deriving a "uniform" steepest descent approximation which is accurate in the simultaneous limit $N \rightarrow \infty, A(N) \rightarrow 0$. For Hermite functions, one finds $\left|z_{s}\right|$ is no longer small in 
comparison to the turning points of the Hermite function, so it is necessary to replace $h_{n}^{ \pm} \sim \exp \left[ \pm i(2 n+1)^{1 / 2} z\right]$ by more accurate WKB approximations to the Hermite functions as done in the next section.

\section{4. "Uniform" AsYmptotics for "SUPER-GausSians"}

When the stationary points $z_{s}$ are $O\left[(2 n+1)^{1 / 2}\right]$. i.e., of the same order of magnitude as the turning points $z_{t}$, it is necessary to replace the regular asymptotic approximations to $h_{n}^{ \pm}$given by (2.24), which apply when $n \rightarrow \infty$ for fixed $z$, with WKB approximations that apply when both $n$ and $z$ are large. If one writes the parabolic cylinder equation in the form

$$
\frac{d^{2} h_{n}}{d z^{2}}+Q(z) h_{n}=0
$$

where

$$
Q(z) \equiv 2 n+1-z^{2}
$$

then

$$
\begin{aligned}
h_{n}^{ \pm} & =[\text {constant }] Q(z)^{-1 / 4} e^{ \pm i P(z)} \\
P(z) & \equiv \int^{z} \sqrt{2 n+1-z^{\prime 2}} d z^{\prime}
\end{aligned}
$$

Boyd [1] showed that for entire functions with suitable rescaling, one could obtain "geometric" convergence-i.e., an exponential index of convergence $r=1$. The same is true for Hermite functions. Define $A$ via

$$
A=\frac{A}{k(2 N+1)^{(k / 2-1)}}
$$

where $N$ is the degree of the highest Hermite function that will be retained in the truncation. With the change of variable

$$
\tilde{z} \equiv z /(2 n+1)^{1 / 2},
$$

the phase function $\phi$ in the steepest descent method can be written (for $h_{N}^{ \pm}$)

$$
\phi=(2 N+1)\left\{-(\Lambda / k) \tilde{z}^{k} \pm i \int^{\tilde{z}} \sqrt{1-z^{\prime 2}} d z^{\prime}\right\}
$$

Thus, the $N$-dependence can be factored out, and the problem of optimizing Hermite convergence for entire functions reduces to that of finding the best $A$, which is a function of $k$ only. 
Numerically, this is trivial, but along the lines of Boyd [1] one can show analytically that writing

$$
\tilde{z_{s}}=\rho e^{i \mu}
$$

one finds

$$
\begin{aligned}
& \mu=\frac{\pi}{2 k} \\
& \rho=\frac{1}{\sqrt{2 \cos (\pi / k)}} \\
& A=1 / \rho^{k-2}
\end{aligned}
$$

Table I shows $\Lambda$ and the real part of $\phi$ divided by $N$ evaluated at the stationary point for various $k$. For large $N$, the algebraic factors of $N$ implicit in the $Q^{-1 / 4}(x)$ in (4.3) and the steepest descent factor of $\left(-\phi^{\prime \prime}\right)^{1 / 2}$-together, they divide $a_{N}$ by $O\left(N^{-1 / 2}\right)$-make only a negligible contribution to the asymptotic ratio of $E_{N} / E_{N+1}$ (where $E=$ absolute error) which is also shown in the table.

Clearly, convergence becomes poorer as one moves away from $k=2$ to larger values. As shown in the next section, convergence also becomes increasingly poor as $k$ moves away from $k=2$ for $k<2$. In the intermediate case, $k=2$ exactly, it is known from examples (the expansion of $\exp \left[-\boldsymbol{A} z^{2}\right]$, whose exact coefficients are known in closed form) that $a_{n} / a_{n+1}$ may be arbitrarily large.

For comparison purposes, the corresponding Chebyshev results ("domain truncation") from Boyd [1] are also given. One sees that Hermite polynomials are superior to Chebyshev polynomials for $k \leqslant 2 \leqslant 5$.

Table II gives selected Hermite coefficients for $\exp \left(-A z^{4}\right)$ for various $A$. The coefficients do not decrease monotonically with $n$ but rather as a damped oscillation. The oscillation is responsible for the large and variable errors in part (b) of the table. This

TABLE I

Optimum Parameters for "Super-Gaussians," as Predicted by the "Uniform Asymptotics of Section 4 ( $k$ is the Order of $f(z)$ )

\begin{tabular}{cccc}
\hline$k$ & $\Lambda$ & $\delta[$ Hermite $]$ & $\delta[$ Chebyshev $]$ \\
\hline 3 & 1.00 & 1.93 & 1.53 \\
4 & 1.43 & 1.55 & 1.49 \\
5 & 2.04 & 1.40 & 1.46 \\
6 & 2.97 & 1.32 & 1.43 \\
\hline
\end{tabular}

For comparison, the ratio $\delta \equiv E_{N} / E_{N+1}$ is given for both Hermite series and for Chebyshev series with the latter taken from [1], where $E_{N} \equiv\left|a_{N}\right|$ is the approximate absolute error (as in [1]) in discarding $h_{N+1}(z)$ and all higher Hermite functions. Is defined by Eq. (4.5). 
TABLE II

Hermite Coefficients for a "Super-Gaussian," $f(z)=\exp \left(-A z^{4}\right)$

\begin{tabular}{|c|c|c|c|c|c|}
\hline$N$ & $A=0.0017$ & $A=0.0087$ & $A=0.0059$ & $A=0.0041$ & $A=0.0035$ \\
\hline 10 & $-5.92 E-3$ & $2.65 E-3$ & $6.24 E-2$ & $1.33 E-1$ & $1.99 E-1$ \\
\hline 20 & $1.44 E-4$ & $-6.91 E-5$ & $1.15 E-4$ & $-3.40 E-4$ & $4.06 E-6$ \\
\hline 30 & $-2.63 E-5$ & $-4.43 E-7$ & $1.21 E-6$ & $-1.58 E-6$ & $1.83 E-6$ \\
\hline 40 & $-1.29 E-7$ & $1.48 E-8$ & $-1.14 E-8$ & $-8.45 E-10$ & $2.84 E-9$ \\
\hline 50 & $1.14 E-8$ & $-8.23 E-11$ & $8.07 E-11$ & $1.78 E-10$ & $-1.52 E-10$ \\
\hline$N$ & \multicolumn{2}{|c|}{$\log \left|a_{N}\right|[$ predicted $]$} & $\log a_{N}$ [actual $]$ & \multicolumn{2}{|c|}{ Relative Error (\%) } \\
\hline 10 & \multicolumn{2}{|c|}{-4.38} & -5.13 & \multicolumn{2}{|r|}{14.6} \\
\hline 20 & \multicolumn{2}{|c|}{-8.77} & -9.58 & \multicolumn{2}{|r|}{8.5} \\
\hline 30 & \multicolumn{2}{|c|}{13.1} & -13.6 & \multicolumn{2}{|r|}{3.7} \\
\hline 40 & \multicolumn{2}{|c|}{-17.5} & -20.9 & \multicolumn{2}{|r|}{16.3} \\
\hline 50 & \multicolumn{2}{|c|}{-21.9} & -22.6 & \multicolumn{2}{|r|}{3.0} \\
\hline
\end{tabular}

In the top table, selected coefficients $a_{N}$ are listed for five different values of $A$, which are optimum according to Eqs. (4.5) and (4.11) for values of $N=10,20,30,40$, and 50, respectively. The five coefficients in boxes should decrease in magnitude like the terms of a geometric series; the lower table compares the logarithms of the predicted and actual $\left|a_{N}\right|$, where the latter are the boxed numbers of the top table.

oscillation is prediction by (3.13), but the optimal formulas of this section ignore it because the phase of the oscillation is much more sensitive to the specific $f(z)$ which is being expanded than is the decreasing amplitude of the coefficients. Nonetheless, the general trend is clearly as predicted. The first column of part (a) uses a value of $A$ which is predicted to be optimal for $N=10$ while the fifth column employs $A_{\text {opt }}(N=50)$. One can clearly see that using $A_{\text {opt }}(N=50)$ produces $\left|a_{50}\right|$ a hundred times smaller than using $A_{\text {opt }}(N=10)$, but $A_{\text {opt }}(N=50)$ is clearly a poor choice when only a few Hermite functions are used because this value also makes $\left|a_{10}\right|$ thirty times larger than the corresponding value in the first column. Varying $A$ with the truncation $N$ is essential for numerical efficiency.

Part (b) of the table shows that by using (4.11), one can make the logarithm of the error increase linearly with $N$-a geometric process. The table also shows that the simple formula

$$
\log \left|a_{n}\right| \sim-N \log \delta
$$

where $\delta(k)$ is a constant given in Table 1 consistently overestimates the error. The reason is that (4.12) incorporates only the exponential dependence of $a_{N}$ on $N$ generated by $\exp \left[-\phi\left(z_{s}\right)\right]$. The full steepest descent evaluation gives an expression of the form

$$
\log \left|a_{N}\right| \sim-N \log \delta(k)-\frac{1}{2} \log N+\log q
$$


where $\log q$ is a constant independent of $N$, but wholly dependent on the specific $f(z)$ which is being expanded. The correction $-\left(\frac{1}{2}\right) \log N$ is independent of $f(z)$-even of the order $k$ of $f(z)$-and arises from the $1 / \sqrt{-\phi^{\prime \prime}\left(z_{s}\right)}$ factor. Equation (4.13) removes most of the error overestimate, but from a practical numerical analysis standpoint, (4.12) is better. It is simpler, and in an actual Galerkin's or pseudospectral solution of a differential equation, one cannot cut the a priori error estimate too close and still obtain a believable result. For an estimate like (4.12) to be a little conservative is not a bad thing.

\section{5. "Sub-Gaussian" Functions}

Naive calculation of the stationary points in the method of steepest descents using the uniform, WKB asymptotic approximations to the Hermite functions show that for "sub-Gaussian" functions, i.e., those with exponent of real axis decay $k<2$,

$$
z_{s} \doteq z_{t} \equiv \pm \sqrt{2 n+1}
$$

In words, the two stationary points for the coefficient integral $a_{n}$ are approximately at the turning points $z_{t}$ where the corresponding $n$th Hermite function makes the transition from oscillatory behavior $\left(|z|<\left|z_{t}\right|\right)$ to monotonic exponential decay. Unfortunately, this is precisely the neighborhood where the WKB approximations are not accurate, but at least one knowns that this region is of decisive importance in evaluating the integral.

A local approximation to the normalized Hermite function in the vicinity of a turning point can be looked up in the "NBS Handbook of Functions" [8] ("Darwin's expansions," specialized to the immediate neighborhood of $z_{t}$ ) to obtain

$$
h_{n}(z) \sim \frac{\lambda}{(2 n+1)^{1 / 4}} A_{i}\left(\lambda\left|z-z_{t}\right|\right)
$$

where

$$
\lambda \equiv 2^{1 / 3}(2 n+1)^{1 / 6}
$$

subject to the restrictions of (i) small $\left|z-z_{t}\right|$ and (ii) $n \gg 1$. Equation (5.2) is valid for both turning points provided that the sign of $\left(z-z_{t}\right)$ is reversed when $z_{t}<0$; for clarity, we shall focus on the positive turning point in the rest of the section.

Outside the turning point, both the Hermite function and $f(z)$ are exponentially decaying. Consequently, the breakdown of the Airy approximation for large $z-z_{t}$ is irrelevant because the region where the Airy approximation is poor makes only an exponentially small contribution to the integrand so that one can safely replace $h_{n}(z)$ by the Airy function for all $z>z_{t}$. Furthermore, $\lambda$ is growing with $n$ so that the Airy function is falling off more and more steeply as $n \rightarrow \infty$. It follows that since $f(z)$ is independent of $n$, it is legitimate to let $f(z) \simeq f(\sqrt{2 n+1})$ for $n \gg 1$ since the Airy 
function will be very small where this approximation breaks down. Therefore, defining

$$
I_{1} \equiv \int_{\sqrt{2 n+1}}^{\infty} f(z) h_{n}(z) d z
$$

one has

$$
\begin{aligned}
& I_{1} \simeq f(\sqrt{2 n+1}) \int_{0}^{\infty} \frac{A_{i}|\lambda x| \lambda d x}{(2 n+1)^{1 / 4}} \\
& I_{1} \simeq \frac{f(\sqrt{2 n+1})}{3(2 n+1)^{1 / 4}}
\end{aligned}
$$

using the known value for the integral of the Airy function.

For $|z|<\left|z_{t}\right|$, both Hermite function and the Airy approximation to it are oscillatory, but one can now exploit the functions $h_{n}^{+}$and $h_{n}^{-}$defined earlier. Figure 2 shows the pattern of "Stokes lines" for the Hermite functions in the complex plane as given in the monograph on WKB theory by J. Heading [10]. Along these curves, the two WKB solutions to the parabolic cylinder equation exponentially grow or decay

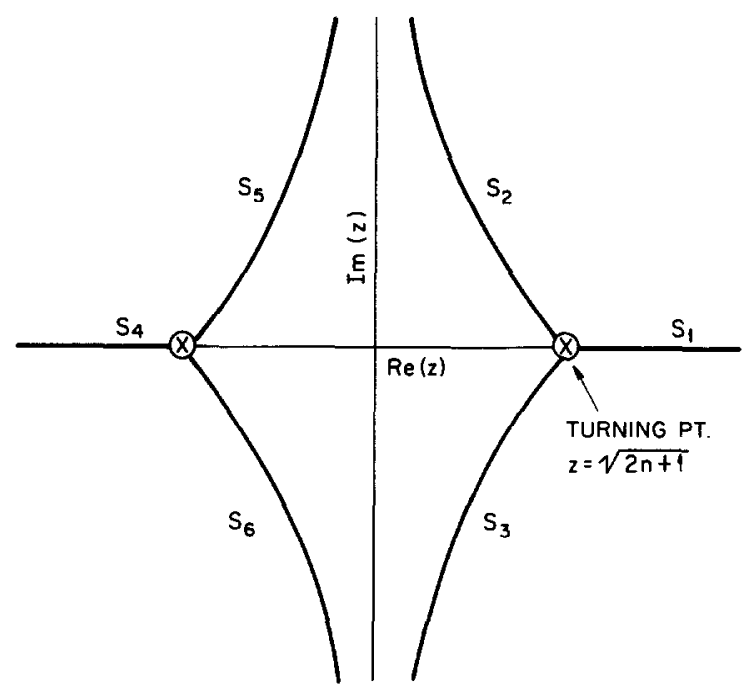

Fig. 2. Steepest descent paths for "sub-Gaussian" functions. These are independent of $f(z)$ [unlike the contours for "super-Gaussians" shown in Fig. 1] and follow the so-called "Stokes lines" of the WKB approximations to the Hermite functions. For $h_{n}^{+}(z)$, the path is $S_{4}-S_{5}-S_{2}-S_{1}$, in that order while for $h_{n}^{-}(z)$ it is the reflection of this path with respect to real $z$ axis, i.e., $S_{4}-S_{6}-S_{3}-S_{1}$. Note that all the Stokes lines extend to infinity. An " $x$ " inside a circle denotes the turning points of $h_{n}^{ \pm}(z)$, $z= \pm(2 n+1)^{1 / 2}$, where three Stokes lines intersect at equal angles of $120^{\circ}$ and also where the Hermite functions $h_{n}(z)$ change from oscillatory to exponentially decaying behavior along the real axis. 
without change of phase. It follows that in the upper half-plane, $h_{n}^{+}$decays exponentially away from the two turning points. In the vicinity of a turning point where the Stokes' curves run together, the WKB approximation breaks down, but it is again possible to match the WKB solution to the appropriate Airy function, which then gives the local approximation in the vicinity of the turning point. "Appropriate" means that the Airy function must decay exponentially away from the turning point along the same Stokes curve as the function it is matched to.

This suggests the decomposition of

$$
J \equiv \int_{-\sqrt{2 n+1}}^{\sqrt{2 n+1}} f(z) h_{n}(z) d z
$$

into the sum

$$
J=I_{2}+I_{3}
$$

where

$$
\begin{aligned}
& I_{2}=\int_{-\sqrt{2 n+1}}^{\sqrt{2 n+1}} f(z) h_{n}^{+}(z) d z \\
& I_{3}=\int_{-\sqrt{2 n+1}}^{\sqrt{2 n+1}} f(z) h_{n}^{-}(z) d z
\end{aligned}
$$

If the path for integration for $I_{2}$ is deformed so as run to $i \infty 0$ along the Stokes line $S_{2}$ (and $S_{5}$ on the other side of the imaginary axis), then this will be a steepest descent path since $h_{n}^{+}(z)$ is decaying exponentially away from both turning points. A similar argument-with the contour of integration deformed into the lower halfplane-applies to $I_{3}$. Then $I_{2}$ and $I_{3}$ can be handled in the same way as $I_{1}$ : by replacing $f(z)$ by its value at the appropriate turning point and by replacing the Hermite function by its local Airy approximation and extending the limits of integration to $\infty$.

The Airy identity [8]

$$
A_{i}(z)=-e^{-2 \pi i / 3} A_{i}\left(z e^{-2 \pi i / 3}\right)-e^{2 \pi i / 3} A_{i}\left(z e^{2 \pi i / 3}\right)
$$

gives the required Airy functions. That on the left of (5.11) matches to $h_{n}(z)$ while the two on the right match to $h_{n}^{+}(z)$ and $h_{n}^{-}(z)$, respectively. Note that the Stokes' curve $S_{2}$ is at angle of $120^{\circ}$, i.e., a rotation through $\exp [2 \pi i / 3]$, relative to the positive real axis so that the first term on the right in (5.11) does indeed decay exponentially on $S_{2}$ as it should. Thus

$$
\begin{aligned}
I_{2} & =\frac{f(\sqrt{2 n+1})}{(2 n+1)^{1 / 4}} \int_{i \infty}^{\sqrt{2 n+1}}-e^{-2 \pi i / 3} \lambda A_{i}\left(\lambda\left[z-z_{t}\right] e^{-2 \pi i / 3}\right) d z+[] \\
& \doteq \frac{f(\sqrt{2 n+1})}{(2 n+1)^{1 / 4}} \int_{0}^{\infty} A_{i}(x) d x+[]
\end{aligned}
$$


where [ ] denotes the contribution from negative $z$ and the other turning point and where (5.12) follows from (5.13) by changing the integration variable to $x=\exp [-2 \pi i / 3] \lambda\left(z-z_{t}\right)$, extending the limit of integration to infinity, and reversing the direction of the integration (away from the turning point instead of towards it, which eliminates the minus signs in (5.11) and (5.12)). Comparing (5.13) with (5.5), one finds that the positive term makes an identical contribution to each of $I_{1}, I_{2}$, and $I_{3}$. Since the definite integral of the Airy function is $\frac{1}{3}$, adding together $I_{1}, I_{2}$, and $I_{3}$ gives finally

$$
a_{n} \sim \frac{f(\sqrt{2 n+1})+f(-\sqrt{2 n+1})}{(2 n+1)^{1 / 4}}
$$

The author proved earlier [7] that the exponential index of convergence $r$ was bounded by $r \leqslant k / 2$, where $k$ is the exponent of real axis decay. Substituting $f(z) \sim$ $\exp \left(-p|z|^{k}\right)$ for $|z| \gg 1$ into (5.14) gives

$$
a_{n} \sim \frac{2}{(2 n+1)^{1 / 4}} e^{-p(2 n+1)^{k / 2}}
$$

which shows that

$$
r=k / 2
$$

exactly for all functions for which this steepest descent argument can be justified.

For "super-Gaussian functions, i.e., $k>2$, the argument given here does not apply because "super-Gaussians" blow up exponentially in certain sectors of the complex plane faster than the $h_{n}^{ \pm}(z)$ decay. This implies that the integrands for $I_{2}$ and $I_{3}$ arc unbounded when deformed away from the real axis onto the Stokes lines.

When $f(z)$ has a singularity, one must add in the residue at the pole; if $k>1$, this residue will far outweigh the turning point contribution and will determine the asymptotic Hermite coefficients. For $k<1$, however, the turning point contribution is exponentially larger than the residue at the pole. In this case, $r<\frac{1}{2}$ so that the Hermite series is convergent only on the real axis even if $f(z)$ is entire and has no singularity at all. For $k=1, r=\frac{1}{2}$ and the strip of convergence is of finite width. If $f(z) \sim \exp (-p|z|)$ and the singularity is a distance $\tau$ from the real $z$-axis, then the width of the strip of convergence is

$$
w=\min (p, \tau)
$$

Equation (5.17) and the fact that the Hermite series of an entire function diverges everywhere except the real axis if $k<1$ were known to Hille [4] although the exact form of the asymptotic coefficients was not. As an illustration of both (5.17) and (5.14), Table III compares the exact Hermite coefficients, found via numerical integration, with those given by (5.14) for $f(z)=\operatorname{sech}(z)$. Though this has poles at $z= \pm i \pi / 2$, the slow decay along the real axis $(\operatorname{sech}(z) \sim \exp (-|z|))$ is the "rate- 
TABLE III

The Hermite Expansion Coefficients of $f(z)=\operatorname{sech}(z)$ and Related Functions

\begin{tabular}{|c|c|c|c|c|c|c|}
\hline$N$ & $a_{n}$ & $a_{n}^{\mathrm{ASYMP}}[$ Eq. 5.14] & $\begin{array}{l}\text { Relative } \\
\text { Error (\%) }\end{array}$ & $b_{n}$ & $\begin{array}{l}\text { Relative } \\
\text { Error (\%) }\end{array}$ & $c_{n}$ \\
\hline 0 & 1.40 & 1.30 & 7.1 & 0.97 & -33 & 0.324 \\
\hline 4 & $1.25 E-1$ & $1.15 E-1$ & 8.0 & $1.05 E-1$ & -9.2 & $1.94 E-2$ \\
\hline 8 & $3.32 E-2$ & $3.19 E-2$ & 4.0 & $3.01 E-2$ & -6.0 & $3.13 E-3$ \\
\hline 12 & $1.23 E-2$ & $1.21 E-2$ & 1.8 & $1.15 E-2$ & -4.6 & $7.46 E-4$ \\
\hline 16 & $5.36 E-3$ & $5.34 E-3$ & 0.4 & $5.14 E-3$ & -3.8 & $2.21 E-4$ \\
\hline 20 & $2.61 E-3$ & $2.62 E-3$ & -0.4 & $2.53 E-3$ & -3.4 & $7.56 E-5$ \\
\hline 24 & $1.37 E-3$ & $1.38 E-3$ & -0.8 & $1.34 E-3$ & -3.0 & $2.86 E-5$ \\
\hline 28 & $7.57 E-4$ & $7.66 E-4$ & -1.1 & $7.46 E-4$ & -2.7 & $1.17 E-5$ \\
\hline 32 & $4.38 E-4$ & $4.44 E-4$ & -1.3 & $4.33 E-4$ & -2.5 & $5.10 E-6$ \\
\hline 36 & $2.63 E-4$ & $2.66 E-4$ & -1.4 & $2.60 E-4$ & -2.4 & $2.34 E-6$ \\
\hline 40 & $1.62 E-4$ & $1.65 E-4$ & -1.5 & $1.61 E-4$ & -2.2 & $1.12 E-6$ \\
\hline 44 & $1.03 E-4$ & $1.04 E-4$ & -1.5 & $1.02 E-4$ & -2.1 & $5.54 E-7$ \\
\hline 48 & $6.63 E-5$ & $6.73 E-5$ & -1.6 & $6.60 E-4$ & -2.0 & $2.82 E-7$ \\
\hline
\end{tabular}

Two competing influences struggle to limit the convergence of sech (z): (i) the poles at $z= \pm i \pi / 2$ and (ii) the slow decay along the real axis, $f(z) \sim O(\exp -|z|)$ for large $|z|$. The real axis decay is stronger, so one can apply (5.14) with good results even though $\operatorname{sech}(z)$ is not an entire function. The nonmonotonic behavior of the relative error with $n$, however, suggests that the poles are not completely negligible for moderate $n$. To confirm this, the coefficients $\left\{b_{n}\right\}$ of $g(z) \equiv \operatorname{sech}(z)-\pi \exp (-0.5$ $\left.\left[z^{2}+\pi^{2} / 4\right]\right) /\left(z^{2}+\pi^{2} / 4\right)$ are also given. This has the same rate of real axis decay as sech $(z)$ (so $b_{n}^{\mathrm{ASYM}}{ }^{\mathrm{P}} \equiv a_{n}^{\mathrm{ASYM}}$ according to (5.14)), but the worst singularities have been subtracted out. The column second from the right shows the errors in the asymptotic approximation decrease monotonically with $n$ now that the poles have been removed. Another perspective is provided by the coefficients $\left\{c_{n}\right\}$ of $t(z) \equiv$ $\operatorname{sech}(z) \exp \left\{-0.5\left(z^{2}+\pi^{2} / 4\right)\right\}$, which has the same poles and same residues as $\operatorname{sech}(z)$, but decays much faster along the real $z$ axis. The $c_{n}$ decrease much more rapidly than the $a_{n}$, showing that the real axis decay causes the relatively slow decrease with $n$ of the coefficients of $\operatorname{sech}(z)$.

determining" factor. The width of the strip of convergence $w=1$, and the coefficients are accurately given by (6.14) alone for large $n$; the contributions of the singularities are asymptotically negligible.

\section{Functions with Singularities}

For functions with poles, one can still apply the method of steepest descent, but since the stationary points are farther and farther from the real axis as $n \rightarrow \infty$, it follows that for sufficiently large $n$, the pole nearest the real axis will be between the steepest descent contour and the original path of integration along the real axis. It follows that the asymptotic Hermite coefficients are the sum of two distinct contributions: (i) the stationary point contributions and (ii) the residues at the poles. When $k>1$, the residues dominate; when $k<1$, the stationary point contributions dominate, and for $k=1$, either contribution may be dominant. 
Hille [3] showed that the Hermite coefficients are $O\left(\exp \left[-\tau(2 n+1)^{1 / 2}\right]\right)$ when $f(z)$ has a singularity (not necessarily a pole) a perpendicular distance $\tau$ from the real $z$ axis. In a moment, complete asymptotic Hermite coefficients will be given for a function whose strip of convergence is determined by a simple pole. First, however, it is useful to prove the following.

LEMMA. If the strips of convergence of two functions $f(z)$ and $g(z)$ are limited by poles at the same location with the same residue, then if $\left\{a_{n}\right\}$ and $\left\{b_{n}\right\}$ are the Hermite coefficients of $f(z)$ and $g(z)$, respectively, then

$$
a_{n} \sim b_{n}
$$

as $n \rightarrow \infty$, with an error which is exponentially small in $n$.

Proof. Under the assumptions given in the lemma,

$$
d(z) \equiv f(z)-g(z)
$$

has a greater strip of convergence in the complex plane than $f(z)$ and $g(z)$. To be precise, let $\tau$ be the absolute value of the imaginary part of the location of that common pole of $f(z)$ and $g(z)$ which is nearest the real axis and let $\sigma$ be the absolute value of that more remote singularity of $d(z)$ which is closest to the real $z$ axis. From Hille's work, letting $\left\{c_{n}\right\}$ denote the expansion coefficients of $d(z)$, it follows that,

$$
\begin{aligned}
& a_{n} \sim p_{1} \exp \left[-(2 n+1)^{1 / 2} \tau\right] \\
& b_{n} \sim p_{2} \exp \left[-(2 n+1)^{1 / 2} \tau\right] \\
& c_{n} \sim p_{3} \exp \left[-(2 n+1)^{1 / 2} \sigma\right]
\end{aligned}
$$

for some constants $p_{1}, p_{2}$, and $p_{3}$. Since $\sigma>\tau$, the only way (6.3)-(6.5) is consistent with $(6.2)$ is if

$$
p_{1}=p_{2}
$$

i.e., the proportionality constants for the asymptotic Hermite coefficients must be the same even if $f(z)$ and $g(z)$ have different orders $k$ of real axis decay.

The usefulness of the theorem is that it shows that if one calculates the asymptotic Hermite coefficients for one function, the same formula applies to all $f(z)$ even if it is not possible to apply the method of steepest descent to all. For a model like

$$
f(z)=\frac{e^{-z^{2}}}{\left(z^{2}+\gamma^{2}\right)}
$$

the steepest descent contours can be calculated explicitly. The results, combined with the lemma, prove the following. 
THEOREM. For a function $f(z)$ whose convergence is limited by simple poles at the roots of $z^{2}=-\gamma^{2}$ with residue $R$, the non-zero expansion coefficients $\left\{a_{n}\right\}$ of $f(z)$ as a series of normalized Hermite polynomials have magnitudes asymptotically given by

$$
\left|a_{n}\right| \sim 2^{5 / 4} \pi^{1 / 2} R n^{-1 / 4} e^{-\gamma(2 n+1) 1 / 2}
$$

Table IV illustrates the accuracy of (6.8) for a particular case.

The extension to higher order poles is trivial; the direct proof of (6.8) and its generalizations is to simply evaluate the residuals of the product $h_{n}^{ \pm}(z) f(z)$ at the convergence-limiting singularities and then add them, assuming $f(z)$ is such that the method of steepest descent can be applied. Since the poles are fixed while the turning points $\left|z_{t}\right|$ of the Hermite functions tend to infinity as $z \rightarrow \infty$, it follows that one can safely use the ordinary asymptotics of $h_{n}^{ \pm},(2.24)$, in evaluating the residuals.

\section{TABLE IV}

Comparison of the Exact and Asymptotic Hermite Coefficients for a Typical Function Whose Series Convergence Is Determined by Its Singularities, $f(z)=\exp \left(-0.5 z^{2}\right) /\left(1+z^{2} / \gamma^{2}\right)$

\begin{tabular}{llllll}
\hline$N$ & $a_{n}^{\text {EXACT }}$ & $a_{n}^{\text {ASYMP }}$ & $\begin{array}{c}\text { Relative } \\
\text { Error }(\%)\end{array}$ & $a_{n}^{\text {IMPROVED }}$ & $\begin{array}{c}\text { Relative } \\
\text { Error (\%) }\end{array}$ \\
\hline & & & $\gamma=\pi / 4$ & \\
\hline 10 & 0.0333 & 0.0347 & 4.1 & 0.0341 & 2.3 \\
20 & 0.00681 & 0.00697 & 2.4 & 0.00689 & 1.1 \\
30 & 0.00204 & 0.00208 & 2.3 & 0.00207 & 1.0 \\
& & & & & \\
\hline 10 & 0.00398 & 0.00478 & 20 & 0.00415 & 4.3 \\
20 & $2.04 E-4$ & $2.30 E-4$ & 13 & $2.08 E-4$ & 1.5 \\
30 & $2.07 E-5$ & $2.28 E-5$ & 10 & $2.10 E-5$ & 1.0 \\
40 & $3.02 E-6$ & $3.28 E-6$ & 8.5 & $3.05 E-6$ & \\
& & & & & 4.6 \\
& & & & & \\
\hline 10 & $8.96 E-5$ & $2.90 E-4$ & 223 & $9.37 E-5$ & 3.6 \\
20 & $3.44 E-7$ & $7.99 E-7$ & 132 & $3.57 E-7$ & 2.8 \\
30 & $4.36 E-9$ & $8.68 E-9$ & 99 & $4.48 E-9$ & 2.2 \\
\hline 40 & $1.06 E-10$ & $1.92 E-10$ & 81 & $1.08 E-10$ & \\
\hline
\end{tabular}

This has simple poles at $z= \pm \gamma i$ with residue $R=0.5 \gamma \exp \left(0.5 \gamma^{2}\right)$. The asymptotic formula is (6.8)

$$
a_{n}^{\mathrm{ASYMP}} \sim(-1)^{n / 2} 2^{5 / 4} \pi^{1 / 2} R n^{-1 / 4} \exp \left[-\gamma(2 n+1)^{1 / 2}\right] .
$$

As the poles are moved farther from the real axis (larger $\gamma$ ), the asymptotic formula has small relative error only for very large $n$. Although even a $100 \%$ error is probably acceptable for purposes of optimizing the Hermite solution of a differential equation, it is still of at least academic interest to show that most of the error can be eliminated by expanding the WKB phase integral for the Hermite functions, $\int^{z}\left(2 n+1-z^{\prime 2}\right)^{1 / 2} d z^{\prime}$, to next order. This gives $a_{n}^{\text {IMPROVED }}=\exp \left\{-\gamma^{3} /\left(6[2 n+1]^{1 / 2}\right)\right\} a_{n}^{\text {ASYMP }}$. 
The theorem only applies to poles, but a singularity of the form $\left(z^{2}+\gamma^{2}\right)^{-j}$ for $1<j<2$ is worse than a simple pole, but less singular than a double pole, so one may expect the convergence of its series to be intermediate between those for simple and double poles. One may conjecture that for

$$
\begin{gathered}
f(z)=\frac{e^{-z^{2}}}{\left(z^{2}+\gamma^{2}\right)^{j}} \\
a_{n} \sim O\left[n^{-3 / 4+j / 2} e^{-(2 n+1)^{1 / 2}}\right]
\end{gathered}
$$

even if $j$ is not an integer, but no formal proof will be attempted here.

As in Boyd [1], optimum accuracy for a given finite number $N$ of Hermite polynomials requires rescaling $f(z)$ in such a way as to balance the stationary point and residual contributions. However, no detailed analysis of how to optimize convergence will be given here.

\section{Analytical Applications of Hermite functions}

The preceding sections have done a thorough job of discussing the asymptotic behavior of the Hermite series for functions which decay exponentially on the real axis. To put this in perspective, it is important to note that Hermite functions are often useful analytically as well as numerically. When this is the case, the numerical analysis discussed may not be applicable because either (i) too few Hermite functions are used for the asymptotic approximations for large $n$ to be accurate or (ii) the Hermite coefficients may decrease only algebraically with $n$. Two examples will be given, one for each possibility.

The first is the quartic anharmonic oscillator of quantum mechanics, which is the problem of finding the eigenvalues $E$ of

$$
\frac{d^{2} v}{d z^{2}}+\left(E-z^{2}-\lambda z^{4}\right) v=0
$$

When the coupling constant $\lambda=0$, the Hermite functions $h_{m}(z)$ are the exact eigenfunctions and $E=2 m+1$. This suggests perturbation theory, but the series for the ground state $[11]$

$$
E_{0}(\lambda)=1+\frac{3}{4} \lambda-\frac{21}{16} \lambda^{2}+\frac{333}{64} \lambda^{3}+\cdots
$$

is asymptotic and diverges for all $\lambda$. For computational purposes, it is useless when $\lambda>0.4$.

Equation (7.1) has therefore been attacked by numerical methods, and the results are a ringing endorsement of the ideas explained in earlier sections. Birkhoff and Fix [12], for example, found that using Galerkin's method with Hermite functions was very sensitive to the choice of scale factor, i.e., to the constant $a$ when $h_{n}(a z)$ are used 
as the spectral basis functions. While $a=1$ gave the first 18 eigenvalues to only three decimal places, (for a particular $\lambda$ ), $a=0.465$ gave the same eigenvalues to 10 decimal places-an increase of $10^{-7}$ in accuracy. The whole purpose of the earlier sections of this paper, of course, is to take the guesswork out of estimating $a$, which Fix and Birkhoff optimized through trial and error.

Banerjee [13] took the scale factor in the form

$$
a=4\left(0.5+B m^{1 / 3} \lambda^{1 / 3}\right)^{1 / 2}, \quad \text { where } m \text {, an integer, is the mode number. }
$$

Since the constant $B$ was found empirically, this formula again involved trial and error. It is, however, more sophisticated than the analysis of Sect. 4 in the sense that the latter would simply identify $v(z)$ as an entire function of order $k=3$, which corresponds to approximating (7.1) as

$$
\frac{d^{2} v}{d z^{2}}-\lambda z^{4} v=0
$$

By the $W K B$ method, the solution of (7.5) has the asymptotic behavior

$$
v \sim[] e^{-\lambda 1 / 2(1 / 3)|z|^{3}}
$$

where the [ ] stands for an irrelevant algebraic function of $z$. In reality, both the eigenvalue $E$ and the unperturbed term $z^{2}$ influence the asymptotic behavior, which for (7.1) is really

$$
v \sim[] e^{-\int z \sqrt{E(m)-z^{2}-\lambda z^{4} d z}}
$$

When $\lambda \ll 1$, the quartic term is important in physical space only for $|z| \gg 1$ and for the Hermite coefficients only for very, very large $n$-perhaps much larger than the chosen truncation of the series. Thus, blindly applying the arguments of Section 4 to (7.1) is reasonable only for large $\lambda$ and small eigenmode number $m$. Banerjee's empirical formula (7.4) is much more uniform in $\lambda$ and $m$.

However, this does not detract from the usefulness of the steepest descent argument presented earlier because one can easily generalize it by using (7.7) instead of (7.6) in asymptotically evaluating the Hermite coefficient integrals. The result will bc more complicated than the simple formulas of Sect. 4 , but it will be a systematic formula rather than an empirical one. In particular, a formula based on steepest descent would allow the scale factor $a$ to vary with the degree $N$ of the truncation of the Hermite series-which (7.4) does not.

These considerations are important, however, only if one is interested in high accuracy. Finlayson [14] has shown that spectral methods with other types of basis functions - Chebyshev polynomials, Fourier series, and so on-can be accurate to within a few percent with as few as two basis functions. The same is true for Hermite functions: Recall that a single Hermite function is the unperturbed or limiting solution for many physical problems as discussed in the introduction. 
The quartic oscillator has coefficients which are symmetric about $z=0$, so its eigenfunctions are all either symmetric about $z=0$ [even modes including the ground state, $m=0$ ] or antisymmetric about $z=0$. Consequently, the computation can be drastically shortened by assuming a series of only even-numbered (symmetric) Hermite functions for the symmetric modes. Using

$$
(a, b)=\int_{-\infty}^{\infty} d z a(z) b(z)
$$

for any two functions $a(z)$ and $b(z)$, letting $L$ denote the linear operator

$$
L \equiv \frac{d^{2}}{d z^{2}}-z^{2}-\lambda z^{4}
$$

and truncating the expansion with just two Hermite functions,

$$
v=a_{0} h_{0}(z)+a_{2} h_{2}(z),
$$

Galerkin's method replaces the differential equation (7.1) with the $2 \times 2$ algebraic eigenvalue problem

$$
\left|\begin{array}{ll}
\left(h_{0}, L h_{0}\right) & \left(h_{0}, L h_{2}\right) \\
\left(h_{2}, L h_{0}\right) & \left(h_{2}, L h_{2}\right)
\end{array}\right|\left|\begin{array}{c}
a_{0} \\
a_{2}
\end{array}\right|=-E\left|\begin{array}{c}
a_{0} \\
a_{1}
\end{array}\right|
$$

which performing the integrals is

$$
\left|\begin{array}{cc}
1+(3 / 4 \lambda)-E & \lambda(3 / 2) \sqrt{2} \\
\lambda(3 / 2) \sqrt{2} & 5+(39 / 4) \lambda-E
\end{array}\right|\left|\begin{array}{c}
a_{0} \\
a_{2}
\end{array}\right|=0
$$

The condition that the determinant of the matrix in (7.12) equals 0 gives a quadratic equation for $E(\lambda)$. Solving this and picking the root for which $E(0)=1$ gives

$$
E_{0}(\lambda)=3+5.25 \lambda-\sqrt{4+18 \lambda+24.75 \lambda^{2}}
$$

Although (7.13) becomes inaccurate for large $\lambda$, it has a wider range of usefulness than the perturbation series (7.2). Equation (7.13) gives $E_{0}(\lambda=1)=1.4126$ versus the exact answer of 1.3924 -an error of only $1.3 \%$. The asymptotic series (7.2), in contrast, is useless for $\lambda \geqslant 0.4$. Galerkin's method has the additional virtue of giving the eigenfunction, too, in the (unnormalized) form

$$
v=h_{0}(z)-\left[\frac{-4-9 \lambda+\sqrt{16+72 \lambda+99 \lambda^{2}}}{3 \lambda \sqrt{2}}\right] h_{2}(z)
$$

Clearly, Hermite function series are useful for much more than brute force number crunching.

This point is reiterated in equatorial oceanography. A number of problems ranging 
from the 1959 paper of Yoshida's [15] to the 1982 note of Cane and Moore [16] have been solved via infinite series of Hermite functions in latitude $y$ whose coefficients decay only algebraically with $n$ because the solutions themselves decay only as inverse powers of $y$-not exponentially with $y$-as $|y| \rightarrow \infty$. Despite their poor convergence, such series have been extremely useful because (i) the coefficients can be determined in analytical closed form and (ii) the convergence can be enormously accelerated by using Abel's summability method, a trick introduced by Moore [17].

Yoshida's steady, wind-driven equatorial jet will serve as an example. For unit wind stress, the east-west current, north-south current, and pressure are, respectively,

$$
\begin{aligned}
& u=-2 \sum_{n=0}^{\infty} \frac{I_{n} h_{2 n}(y)}{(4 n+3)(4 n-1)} \\
& v=-2 \sum_{n=0}^{\infty} \frac{\sqrt{n+1 / 2}}{4 n+3} I_{n} h_{2 n+1}(y) \\
& p=-\sum_{n=0}^{\infty} \frac{(4 n+1)}{(4 n+3)(4 n-1)} I_{n} h_{2 n}(y)
\end{aligned}
$$

where

$$
I_{n}=\frac{2^{1 / 2} \pi^{1 / 4}}{2^{n} n !} \sqrt{(2 n) !}
$$

are the Hermite coefficients of 1 , i.e.,

$$
1=\sum_{n=0}^{\infty} I_{n} h_{2 n}(y)
$$

The asymptotic, large $|y|$ behavior of $u$, $v$, and $p$ can be deduced from their governing equations. Letting $\left\{u_{n}\right\},\left\{v_{n}\right\}$, and $\left\{p_{n}\right\}$ denote the Hermite coefficients in (7.15) through (7.19), one finds the following relationship between the asymptotic Hermite coefficients and the asymptotic behavior of the function:

$$
\begin{aligned}
& 1 \sim 1 \quad \text { for }|y| \gg 1 ; \quad I_{n} \sim O\left(n^{-1 / 4}\right) \\
& v(y) \sim 1 / y \quad \text { for }|y| \gg 1 ; \quad v_{n} \sim O\left(n^{-3 / 4}\right) \\
& p(y) \sim 1 / y^{2} \quad \text { for } \quad|y| \gg 1 ; \quad p_{n} \sim O\left(n^{-5 / 4}\right) \\
& u(y) \sim 1 / y^{4} \quad \text { for } \quad|y| \gg 1 ; \quad u_{n} \sim O\left(n^{-9 / 4}\right)
\end{aligned}
$$

The integration-by-parts argument of Section 2 correctly predicts that each additional inverse power of $y$ adds an additional factor of $1 / \sqrt{n}$ to the asymptotic decay of the Hermite coefficients, but it completely misses the common factor of $n^{-1 / 4}$ in (7.20)-(7.23) and underestimates the rate of decay by $O\left(n^{-5 / 4}\right)$. Clearly, something better is needed; Bain [18] gives some interesting new results for Hermite coefficients with finite algebraic order of convergence. 


\section{Summary}

The author's earlier work [1] on applying Chebyshev polynomials on an unbounded domain is here extended to Hermite functions. Naively, one might suppose that Hermite functions are "obviously" superior to Chebyshev polynomials because use of the latter (on an infinite or semi-infinite interval) requires introducing a parameter $L$, which is either the width of the computational domain or a mapping parameter, while the Hermite functions are well defined without such a parameter since their natural domain of orthogonality is $[-\infty, \infty]$. One major theme of this work, however, is that this is a mirage. To obtain good results with series of Hermite functions, one must rescale either $f(z)$ or equivalently, the argument of the Hermite functions, in a manner given by the explicit formulas above. For entire functions (or functions that decay as slowly with $z$ as sech $(z)$ ), the rescaling depends upon $k$, the "order of real axis decay," and upon the truncation of the Hermite series. For singular functions which decay with sufficient rapidity (i.e., have $k>1$ ), the location of the singularity nearest the real axis is also important. As is also true for Chebyshev series, the best scaling parameter for a given function $f(z)$ when 10 Hermite functions are used is different from that which is most efficient when 40 degrees of freedom are kept.

A noteworthy difference from the analogous Chebyshev results is that the Hermite theory for entire functions splits into two cases: "super-Gaussian functions" $(k>2)$ which decay more rapidly than $\exp \left(-A z^{2}\right)$ for any constant $A$, and "sub-Gaussian functions" which decay more slowly than any Gaussian function. The "superGaussian" series can be analyzed by applying the method of steepest descents to the integrals that define the coefficients of the Hermite series, just as for the Chebyshev casc. However, the "sub-Gaussians" require a different technique exploiting the fact that Hermite functions in the vicinity of their turning points-which is the neighborhood that dominates the coefficient integrals for "sub-Gaussian" functions $f(z)$-can be approximated by Airy functions. For "sub-Gaussians," it has not yet been possible to find "uniform" approximations to the Hermite coefficients which remain accurate when $f(z)$ is rescaled in an $N$-dependent way where $N$ is the degree of the highest retained Hermite coefficient.

For singular functions, this work gives the first complete asymptotic expression for the Hermite coefficients of a function whose series convergence is limited by simple poles. As in the Chebyshev case, the asymptotic coefficients are the sum of a steepest descent term (which is identical with that for an entire function with the same order of real axis decay) plus a term proportional to the residue of the pole. Here again, however, the Hermite theory splits into two cases. For Chebyshev polynomials, the convergence of the series for a singular function is always (unless $f(z)$ is rescaled in an $N$-dependent way) determined entirely by the location and residue of the singularity. For Hermite series, this is true only if the order of real axis decay is greater than 1; if $k<1$, the steepest descent term will dominante the residuc term for large $n$. For a function like sech $(z)$ for which $k=1$, both terms may be important, as illustrated in Table III. 
From a mathematical viewpoint, these results have an appealing unexpectedness and elegance. What is appealing from a numerical standpoint is that Table I shows that Hermite series are more efficient than Chebyshev series for at least some classes of functions.

A confession is needed here: The author originally began to think about the asymptotic behavior of Hermite function series in hopes of understanding why early numerical experiments with them worked so poorly in comparison to what one is used to with Chebyshev series for problems on a finite interval. In part, the answer is that problems in an unbounded domain are harder than on a finite interval; Chebyshev methods on $[-\infty, \infty]$ require many polynomials, too. A more important reason is that all Galerkin methods on an infinite interval are sensitive to the choice of scaling or map parameter. With Chebyshev methods, this scaling is explicit in the parameter $L$ of the author's earlier paper [1]; with Hermite methods, it is implicit. Some choice of scaling, however, is inevitable, and everything hinges upon it. A poor choice of scaling was what frustrated the author's early numerical experiments with Hermite series.

A pure mathematician would argue that $\exp \left(-z^{4}\right)$ and $\exp \left(-10 z^{4}\right)$ are the same function, but it is shown here that their Hermite scries converge at wildly different rates. All representations of a given function are not equal; the purpose of this paper is to give some guidance on the one which is most efficient, assuming that one knows something about (i) the asymptotic behavior of the solution $f(z)$ along the real $z$ axis and (ii) where $f(z)$ is singular.

This paper has concentrated upon asymptotic results as $n \rightarrow \infty$ for $f(z)$ which decay exponentially fast as $|z| \rightarrow \infty$. To avoid creating the impression that all practical uses of Hermite functions are covered by these results, Section 7 discusses use of very low order truncations to obtain analytical rather than numerical results and also some problems in oceanography that have been solved via Hermite series for functions which decay algebraically for large $z$.

Several problems remain for the future. One is to improve the results given here for "sub-Gaussians": is it possible to rescale them with $N$ in such a way as to obtain geometric convergence, as is possible for "super-Gaussians" and for Chebyshev methods? A second is to tighten existing results for functions which decay as some finite inverse power of $z$ for large $|z|$. A theorem proved in Sect. 2 shows that the Hermite coefficients must decrease algebraically rather than exponentially with $n$, but both that theorem and Bain's stronger result [18] are far too pessimistic for the oceanographic examples given in Section 7. A third is to look more closely into Hermite functions for problems in spherical geometry.

Hermite functions-on-a-sphere requires some explanation, but Hermite functions are the limiting eigenfunctions of the prolate spheroidal wave equation and also of the spherical wave equations in geophysical fluid dynamics [19]. By transforming into so-called "Mercator" coordinates via

$$
\sin [\phi]=\tanh y
$$

where $\phi$ is latitude and $y$ is the new coordinate, the finite interval in latitude is 
mapped into $[-\infty, \infty]$. One can show that the eigenfunctions of zonal wavenumber $s$ must decay in $y$ like exp $[-s y]$. The complication, which shall not be addressed here, is that this asymptotic behavior is reached only for rather large $|y|$ in the parameter regime in which the waves are "trapped" at low latitudes and are well approximated by a single Hermite function. A full treatment would require applying the ideas of Section 6 of this paper to the $W K B$ approximations to the prolate spheroidal eigenfunctions. The problem may someday need to be solved because such Hermite-like modes are part of the basis for so-called "Hough function" models for numerically forecasting the weather [19].

\section{ACKNOWLEDGMENT}

This work was supported by the National Science Foundation through Grant OCE8108530.

\section{REFERENCES}

1. J. P. Boyd, J. Comp. Phys. 45 (1982), 45-79.

2. D. Gotruleg AND S. A. ORSzag, "Numerical Analysis of Spectral Methods: Theory and Applications," Soc. Ind. and Appl. Math., Philadelphia, 1977.

3. E. Hille, Duke Math. J. 5 (1939), 875-936.

4. E. Hille, Trans. Amer. Math. Soc. (1940), 80-94.

5. E. Hille, Duke Math. J. 7 (1940), 458-495.

6. E. Hille, J. Math. Pure Appl. 40 (1961), 335-342.

7. J. P. Boyd, Math. Comp. 35 (1980), 1309-1316.

8. M. Abramowitz and I. Stegun, "Handbook of Mathematical Functions," Dover, New York, 1965.

9. G. N. Watson, Proc. London Math. Soc. (2) 8 (1910), 393-421.

10. J. Heading, "An Introduction to Phase-Integrals," Wiley, New York, 1977.

11. J. P. Boyd, J. Math. Phys. 19 (1978), 1445-1456.

12. G. BIRkofF AND G. FIX, in "Numerical Solution of Field Problems in Continuum Mechanics," Vol. 2, SIAM-AMS Proceedings, pp. 111-151, Amer. Math. Soc., Providence, 1970.

13. K. BanerueE, Proc. Roy. Soc. London, Ser. A 364 (1978), 265.

14. B. A. Finlayson, "The Method of Weighted Residuals and Variational Principles," Academic Press, New York, 1972.

15. K. Yoshida, J. Oceangr. Soc. Japan 15 (1969), 154-170.

16. M. A. CANe AND D. W. Moore, J. Phys. Oceangr. 11 (1982), 1578-1584.

17. D. W. MOORE, "Planetary-Gravity Waves in an Equatorial Ocean," Ph. D. Thesis, Harvard University, Cambridge, Mass. 1968.

18. M. BaIN, J. Inst. Maths. Appl. 21 (1978), 379-386.

19. A. Kasahara, J. Atmos. Sci. 34 (1977), 687. 